\title{
The essence of the homotopy analysis method
}

\author{
Cheng-shi Liu \\ Department of Mathematics \\ Daqing Petroleum Institute \\ Daqing 163318, China \\ Email: chengshiliu-68@126.com \\ Tel:+86-459-6503476
}

June 1, 2011

\begin{abstract}
The generalized Taylor expansion including a secret auxiliary parameter $h$ which can control and adjust the convergence region of the series is the foundation of the homotopy analysis method proposed by Liao. The secret of $h$ can't be understood in the frame of the homotopy analysis method. This is a serious shortcoming of Liao's method. We solve the problem. Through a detailed study of a simple example, we show that the generalized Taylor expansion is just the usual Taylor's expansion at different point $t_{1}$. We prove that there is a relationship between $h$ and $t_{1}$, which reveals the meaning of $h$ and the essence of the homotopy analysis method. As an important example, we study the series solution of the Blasius equation. Using the series expansion method at different points, we obtain the same result with liao's solution given by the homotopy analysis method.

Keywords: homotopy analysis method; generalized Taylor expansion; series expansion method; nonlinear differential equation; Blasius equation
\end{abstract}

\section{Introduction}

In a series of papers[1-12], Liao developed and applied the homotopy analysis method to deal with a lot of nonlinear problems. In 2004, Liao published the book[13] in which he summarized the basic ideas of the homotopy analysis method and gave the details of his approach both in the theory and on a large number of practical examples. The key of his method is the generalized Taylor expansion. We next describe this result. For a given nonlinear differential equation with initial conditions at the point $t_{0}$, we can obtain the power series solution

$$
f(t)=\sum_{n=0}^{+\infty} \frac{f^{(n)}\left(t_{0}\right)}{n !}\left(t-t_{0}\right)^{n}
$$


with the convergence region $\left|t-t_{0}\right|<\rho_{0}$. Through introducing an approaching function, Liao gives the following so-called generalized Taylor series solution to the nonlinear equation considered,

$$
f(t)=\lim _{m \rightarrow \infty} \sum_{n=0}^{m} \mu_{m, n}(h) \frac{f^{(n)}\left(t_{0}\right)}{n !}\left(t-t_{0}\right)^{n}
$$

where the approaching function $\mu_{m, n}(h)$ satisfies $\lim _{m \rightarrow \infty} \mu_{m, n}(h)=1$ when $n \geq 1$. Liao points out that the generalized Taylor series provides a way to control and adjust the convergence region through an auxiliary parameter $h$ such that the homotopy analysis method is particularly suitable for problems with strong nonlinearity[3]. However, the mathematical meaning of the parameter $h$ is still unknown. This is a serious shortcoming of the homotopy analysis method.

In the present paper, we point out that the so-called generalized Taylor series at the initial point $t_{0}$ is just the usual Taylor expansion of $f(t)$ at another point. Our results give the meaning of $h$ in the generalized Taylor expansion, and also uncover the essence of the homotopy analysis method. We study two examples in detail. Especially, using the series expansion method to solve the Blasius equation, we give the same result with the Liao's generalized Taylor series solution by the homotopy analysis method.

\section{The detailed analysis of a simple example}

In the book [13], Liao had studied a simple example $f(t)=\frac{1}{1+t}$ to illustrate the generalized Taylor series. He gave the following generalized Taylor expansion,

$$
f(t)=\lim _{m \rightarrow \infty} \sum_{n=0}^{m} \mu_{0}^{m, n}(h)\left[(-1)^{n} t^{n}\right]
$$

with the convergence region

$$
-1<t<\frac{2}{|h|}-1,(-2<h<0)
$$

where

$$
\mu_{0}^{m, n}(h)=(-h)^{n+1} \sum_{k=0}^{m-n}\left(\begin{array}{c}
n+k \\
n
\end{array}\right)(1+h)^{k} .
$$

Liao concludes that the series (3) has enlarged the convergence region of the Taylor series of $f$ at the point $t=0$ and calls it the generalized Taylor series.

We next analyze the essence of the generalized Taylor expansion in detail. We prove that the above generalized Taylor series (3) is just the usual Taylor expansion at the point $t_{0}=-1-\frac{1}{h}$. Indeed, the Taylor series of $f$ at the point $t=0$ is

$$
f(t)=1-t+\cdots+(-1)^{n} t^{n}+\cdots,
$$


with the convergence region $|t|<1$. The Taylor series at the point $t_{0}$ is

$$
f(t)=\frac{1}{1+t_{0}}\left\{1-\frac{t-t_{0}}{1+t_{0}}+\cdots+(-1)^{n}\left(\frac{t-t_{0}}{1+t_{0}}\right)^{n}+\cdots\right\}
$$

with the convergence region $\left|t-t_{0}\right|<\left|1+t_{0}\right|$. If we take $t_{0}=-1-\frac{1}{h}$, the above expression (7) becomes

$$
\begin{array}{r}
f(t)=-h\left\{1+h\left(t+1+\frac{1}{h}\right)+\cdots+(-h)^{n}\left(t+1+\frac{1}{h}\right)^{n}+\cdots\right\} \\
=-h \sum_{n=0}^{+\infty}(-1)^{n} \sum_{k=0}^{n}\left(\begin{array}{l}
n \\
k
\end{array}\right) t^{k}(1+h)^{n-k}(-1)^{n} h^{k} \\
=\sum_{k=0}^{+\infty}(-1)^{k} t^{k} \sum_{n=k}^{+\infty}\left(\begin{array}{l}
n \\
k
\end{array}\right)(1+h)^{n-k}(-h)^{k+1},
\end{array}
$$

which is just the generalized Taylor series (3).

When $t_{0}>-\frac{1}{2}$, that is, $-2<h<0$, we have

$$
\lim _{m \rightarrow \infty} \mu_{m, n}(h)=\lim _{m \rightarrow \infty} \frac{1}{\left(1+t_{0}\right)^{k+1}} \sum_{n=k}^{m}\left(\begin{array}{l}
n \\
k
\end{array}\right)\left(-t_{0}\right)^{n-k}=1 .
$$

Correspondingly, the convergence region $\left|t-t_{0}\right|<\left|1+t_{0}\right|$ is just (4).

When $-1 \leq t_{0}<-\frac{1}{2}$ or $t_{0}<-1$, we have

$$
\lim _{m \rightarrow \infty} \mu_{m, n}(h)=\lim _{m \rightarrow \infty} \frac{1}{\left(1+t_{0}\right)^{k+1}} \sum_{n=k}^{m}\left(\begin{array}{l}
n \\
k
\end{array}\right)\left(-t_{0}\right)^{n-k}=\infty .
$$

Therefore, when $t_{0}>-\frac{1}{2}$ and $-1<t<1+2 t_{0}$, that is, $-2<h<0$ and $-1<t<-1-\frac{2}{h}=\frac{2}{|h|}-1$, the right side of expression (3) is just the Taylor series of $f$ at the point $t_{0}=-1-\frac{1}{h}$. From our discussion and computation, it is easy to see that as the Taylor series of $f$ at the point $t_{0}$ the convergence region of the series (3) depends on the point $t_{0}$. When $t_{0} \rightarrow+\infty$, that is, $h \rightarrow 0$, the convergence region $\left(-1,2 t_{0}+1\right)$ of the series $(3)$ becomes $(-1,+\infty)$ naturally. In other words, $t_{0}$ can be used to control and adjust the convergence region.

Our result gives the meaning of the auxiliary parameter $h$, and hence uncovers the essence of the generalized Taylor expansion as the kernel of the homotopy analysis method. In general, we have the following conclusion: the generalized Taylor series at the initial point $t_{0}$ is only the usual Taylor expansion of $f(t)$ at another point $t_{1}=\left(\eta_{0}-t_{0}\right)\left(1+\frac{1}{h}\right)+t_{0}$, where the real number $\eta_{0}$ satisfies $\left|\eta_{0}-t_{1}\right|=\left|\xi_{0}-t_{1}\right|$, and $\xi_{0}$ is the nearest singular point of $f(t)$ from $t_{1}$. Indeed, let $\xi_{k}(k=1,2, \cdots)$ be all singular points of a function $f$ which is an analytic function at the point $t_{1}$ and the convergence radius be $\rho_{0}=\inf \left(\left|t_{1}-\xi_{k}\right|, k=1,2, \cdots\right)$. When $t_{0}$ belongs to the convergence region of the Taylor expansion of $f$ at the point $t_{1}$, we can represent the Taylor series at the point $t_{1}$ by the Taylor series at the point $t_{0}$ as follows,

$$
f(t)=\sum_{n=0}^{+\infty} \frac{f^{(n)}\left(t_{1}\right)}{n !}\left(t-t_{1}\right)^{n}=\sum_{n=0}^{+\infty} \sum_{k=0}^{n} \frac{f^{(n)}\left(t_{1}\right)}{n !}\left(\begin{array}{l}
n \\
k
\end{array}\right)\left(t-t_{0}\right)^{k}\left(t_{0}-t_{1}\right)^{n-k}
$$




$$
\begin{aligned}
& =\sum_{n=0}^{+\infty} \sum_{k=n}^{+\infty} \frac{f^{(k)}\left(t_{1}\right)}{k !}\left(\begin{array}{l}
k \\
n
\end{array}\right)\left(t_{0}-t_{1}\right)^{k-n}\left(t-t_{0}\right)^{n} \\
& =\lim _{m \rightarrow \infty} \sum_{n=0}^{m} \mu_{m, n}\left(f ; t_{0}, t_{1}\right) \frac{f^{(n)}\left(t_{0}\right)}{n !}\left(t-t_{0}\right)^{n},
\end{aligned}
$$

where the approaching function

$$
\mu_{m, n}\left(f ; t_{0}, t_{1}\right)=\left\{\sum_{k=n}^{m} \frac{f^{(k)}\left(t_{1}\right)}{k !}\left(\begin{array}{l}
k \\
n
\end{array}\right)\left(t_{0}-t_{1}\right)^{k-n}\right\} / \frac{f^{(n)}\left(t_{0}\right)}{n !}
$$

satisfies $\lim _{m \rightarrow \infty} \mu_{m, n}\left(f ; t_{0}, t_{1}\right)=1$. Expression (11) means that the usual Taylor expansion at the point $t_{1}$ can be represented by the so-called generalized Taylor expansion at the point $t_{0}$. Let $\xi_{0}$ be the nearest singular point from $t_{1}$ and $h=\frac{\xi_{0}-t_{0}}{t_{1}-\xi_{0}}$, respectively, we have $t_{1}=\left(\xi_{0}-t_{0}\right)\left(1+\frac{1}{h}\right)+t_{0}$. Then the convergence region $\left|t-t_{1}\right|<\left|t_{1}-\xi_{0}\right|$ becomes

$$
\left|1+h-h \frac{t-t_{0}}{\xi_{0}-t_{0}}\right|<1 .
$$

It is just the convergence region of another generalized Taylor series given by Liao in Ref.[14] where the corresponding approaching function is $\mu_{m, n}(h)=$ $(-h)^{n} \sum_{k=0}^{m-n}\left(\begin{array}{c}k+n-1 \\ k\end{array}\right)(1+h)^{k}$ which doesn't depends on $f$. This means that these two series have the same convergence region, and hence our Taylor expansion is equivalent to the Liao's generalized Taylor expansion. If $\xi_{0}$ is not a real number, let $h=\frac{\eta_{0}-t_{0}}{t_{1}-\eta_{0}}$ respectively we have $t_{1}=\left(\eta_{0}-t_{0}\right)\left(1+\frac{1}{h}\right)+t_{0}$ where the real number $\eta_{0}$ satisfies $\left|t_{1}-\xi_{0}\right|=\left|t_{1}-\eta_{0}\right|$. Then the convergence region $\left|t-t_{1}\right|<\left|t_{1}-\xi_{0}\right|$ becomes

$$
\left|1+h-h \frac{t-t_{0}}{\eta_{0}-t_{0}}\right|<1 .
$$

For example, we take $f(t)=\frac{1}{1+t^{2}}$ and $t_{0}=0$. Therefore, we have $\xi_{0}= \pm \mathrm{i}$ and $\eta_{0}=t_{1} \pm \sqrt{t_{1}^{2}+1}$. In order that $t_{0}$ belongs to the convergence region of $f$ at the point $t_{1}$, when $t_{1}>0$ or $t_{1}<0$, we have $\eta_{0}=t_{1}-\sqrt{t_{1}^{2}+1}$ or $\eta_{0}=t_{1}+\sqrt{t_{1}^{2}+1}$ respectively.

Remark. According to different choice of the function $f$ and the points $t_{0}$ and $t_{1}$, we can construct infinite number of approaching functions. For every approaching function, we can give a kind of generalized Taylor expansion method. Therefore there exist infinite number of generalized Taylor expansion methods. In his book[13], Liao gives two kinds of generalized Taylor expansion methods.

\section{On the series solution of the Blasius equation}

Blasius equation reads

$$
f^{\prime \prime \prime}(\eta)+\frac{1}{2} f(\eta) f^{\prime \prime}(\eta)=0,
$$


with the conditions

$$
f(0)=f^{\prime}(0)=0, \quad f^{\prime}(+\infty)=1,
$$

which describes the two-dimensional viscous laminar flow over an infinite flatplain[15]. In 1908, Blasius [15] gave a series solution

$$
f(\eta)=\sum_{k=0}^{+\infty}\left(-\frac{1}{2}\right)^{k} \frac{A_{k} \sigma^{k+1}}{(3 k+2) !} \eta^{3 k+2},
$$

where $\sigma=f^{\prime \prime}(0)$ and

$$
A_{0}=A_{1}=1, \quad A_{k}=\sum_{r=0}^{k-1}\left(\begin{array}{c}
3 k-1 \\
3 r
\end{array}\right) A_{r} A_{k-r-1},(2 \leq k) .
$$

Blasius obtained $\sigma \approx 0.332$ and the convergence region $0 \leq \eta<\rho_{0} \approx 5.690$. In 1997, Liao obtained a generalized Taylor series solution by means of the homotopy analysis method,

$$
f(\eta)=\lim _{m \rightarrow \infty} \sum_{k=0}^{m}\left[\left(-\frac{1}{2}\right)^{k} \frac{A_{k} \sigma^{k+1}}{(3 k+2) !} \eta^{3 k+2}\right] \mu_{0}^{m, k}(h),
$$

which converges in the region

$$
-\rho_{0} \leq \eta \leq \rho_{0}\left[\frac{2}{|h|}-1\right]^{1 / 3},
$$

and where

$$
\mu_{0}^{m, n}(h)=(-h)^{n} \sum_{k=0}^{m-n}\left(\begin{array}{c}
n-1+k \\
k
\end{array}\right)(1+h)^{k} .
$$

Now we use the Taylor expansion of $f(\eta)$ at the point $\eta_{0}$ to obtain the Liao's result. Assuming that

$$
f(\eta)=\sum_{n=0}^{+\infty} a_{n}\left(\eta-\eta_{0}\right)^{n}
$$

and substituting it into the Blasius equation, we have

$$
a_{n+3}=-\frac{1}{2(n+3)(n+2)(n+1)} \sum_{m=0}^{n}(m+2)(m+1) a_{m+2} a_{n-m}, \quad(n \geq 0) .
$$

In order to determine the values of $a_{0}, a_{1}$ and $a_{2}$, we use the conditions (16) to give

$$
\begin{gathered}
\sum_{n=0}^{+\infty} a_{n}\left(-\eta_{0}\right)^{n}=0, \\
\sum_{n=0}^{+\infty}(n+1) a_{n+1}\left(-\eta_{0}\right)^{n}=0,
\end{gathered}
$$


and

$$
\lim _{\eta \rightarrow+\infty} \sum_{n=0}^{+\infty}(n+1) a_{n+1}\left(\eta-\eta_{0}\right)^{n}=1,
$$

the condition (26) also can be replaced by using $\sigma$

$$
\sum_{n=0}^{+\infty}(n+2)(n+1) a_{n+2}\left(-\eta_{0}\right)^{n}=\sigma \approx 0.332 .
$$

Then we can obtain the values of $a_{n}$ for $n=0,1, \cdots$.

If we take

$$
\eta_{0}=\rho_{0}\left[\frac{2}{|h|}-1\right]^{1 / 3} \frac{1+h}{2+h}
$$

we find that the Liao's generalized Taylor series solution is just the usual Taylor series solution at the point $\eta_{0}$. In fact, the convergence region of our series solution is $\eta_{0}-\left|\eta_{0}-\varsigma_{0}\right|<\eta<\eta_{0}+\left|\eta_{0}-\varsigma_{0}\right|$, where the real number $\varsigma_{0}$ satisfies $\left|\eta_{0}-\varsigma_{0}\right|=\left|\eta_{0}-\xi_{0}\right|, \xi_{0}$ is the nearest singular point from $\eta_{0}$. Of course, we don't know the value of $\xi_{0}$. Therefore, our computation is an approximation treatment. Since $\eta=0$ belongs to the region, we require $\varsigma_{0}<0$. Furthermore, let

$$
\eta_{0}=\varsigma_{0}\left(1+\frac{1}{h}\right),
$$

and $\eta_{0}>0$. By assuming that the convergence regions of two series solutions are the same one, we take $\eta_{0}+\left|\eta_{0}-\varsigma_{0}\right|=\rho_{0}\left[\frac{2}{|h|}-1\right]^{1 / 3}$ to give

$$
\frac{(h+2) \eta_{0}}{h+1}=\rho_{0}\left[\frac{2}{|h|}-1\right]^{1 / 3},
$$

from which we obtain the result (28).

For example, we take $h=-\frac{1}{2}$. Correspondingly, we have $\eta_{0} \approx 2.735$. The numerical result is coincident with Liao's[3].

\section{Conclusion}

Through detailed analysis of some examples, we show that the generalized Taylor series is only the usual Taylor expansion at another point. This means that we can use the series expansion at other point to give the same result obtained by the homotopy analysis method. Our results uncover the essence of the generalized Taylor expansion as the key of the homotopy analysis method.

Acknowledgments. I would like to thank the referees for their valuable suggestions.

\section{References}

[1] S. J. Liao, Proposed homotopy analysis techniques for the solution of nonlinear problems, Ph.D. dissertation, Shanghai Jiao Tong University, Shanghai, 1992. 
[2] S.J. Liao, An approximate solution technique which does not depend upon small parameters: a special example, Int. J. Non-linear Mech. 30:371$380(1995)$.

[3] S. J. Liao, An approximate solution technique which does not depend upon small parameters (Part 2): an application in fluid mechanics, Int. J. Nonlinear Mech. 32:815-822(1997).

[4] S. J. Liao, An explicit, totally analytic approximation of Blasius viscous flow problem, Int. J. Non-Linear Mech. 34:759-778(1999).

[5] S. J. Liao, A uniformly valid analytic solution of 2D viscous flow past a semi-infinite flat plate. J. Fluid Mech. 385:101-128(1999).

[6] S.J. Liao, A. Campo, Analytic solutions of the temperature distribution in Blasius viscous flow problems, J.Fluid Mech. 453:411-425(2002).

[7] S. J. Liao, An explicit analytic solution to the Thomas-Fermi equation, Appl. Math. Comput. 144: 495-506(2003).

[8] S.J. Liao, On the analytic solution of magnetohydrodynamic flows of nonNewtonian fluids over a stretching sheet, J. Fluid Mech. 488:189-212(2003)

[9] S. J. Liao, On the homotopy analysis method for nonlinear problems, Appl. Math. Comput. 147: 499-513(2004) .

[10] S.J. Liao, E. Magyari, Exponentially decaying boundary layers as limiting cases of families of algebraically decaying ones, Z. Angew. Math. Phys. 57:777-792(2006).

[11] S.J. Liao, Y. Tan, A general approach to obtain series solutions of nonlinear differential equations, Stud. Appl. Math. 119:297-354(2007).

[12] S.J. Liao, A general approach to get series solution of non-similarity boundary-layer flows, Commun. Nonlinear Sci. Numer. Simulat. 14:21442159(2009).

[13] S. J. Liao, Beyond perturbation: Introduction to the homotopy analysis method, CRC press LLC, Boca Raton, 2003.

[14] S. J. Liao, On a generalized Taylor theorem: a rational proof of the validity of the so-called homotopy analysis method. J. Appl. Math. Mech. 24:47$54(2003)$.

[15] H. Blasius, Grenzschichten in flussigkeiten mit kleiner reibung, Z. Math. Phys. 56:1-37(1908). 